\title{
CAPÍTULO 13: INFLUÊNCIA DA TEMPERATURA DE SECAGEM NAS CARACTERÍSTICAS FÍSICO-QUÍMICAS E NUTRICIONAIS DO BAGAÇO DE CERVEJA
}

\section{CHAPTER 13: INFLUENCE OF DRYING TEMPERATURE ON PHYSICAL- CHEMICAL AND NUTRITIONAL CHARACTERISTICS OF BREWERY BAGACE}

\author{
Lisangela Bagatini ${ }^{1}$, Gabriela Diersmann Azevedo ${ }^{2}$, Voltaire Sant'Anna ${ }^{3}$
}

\section{Resumo}

O bagaço de malte, considerado o maior resíduo sólido cervejeiro é conhecido por conter ricos componentes que permanecem inexplorados durante o processamento. Tendo em vista seu alto teor de umidade, este estudo avaliou os efeitos da secagem sobre características tecnológicas e nutricionais importantes para a indústria alimentícia. Avaliou-se a influência do processo de secagem por convecção natural, entre 60 e $100{ }^{\circ} \mathrm{C}$, na concentração de polifenóis totais, flavonóis, ésteres tartáricos, compostos com atividade antioxidante, $\mathrm{pH}$, cor e capacidade de retenção de água e óleo do bagaço de malte proveniente do processo de produção da cerveja Pilsen. Amostras liofilizadas foram usadas para comparação. Amostras secas à $60{ }^{\circ} \mathrm{C}$ e $80^{\circ} \mathrm{C}$ apresentaram maior concentração de polifenóis totais, flavonóis, ácidos fenólicos e compostos com capacidade de eliminar radicais livres. $\mathrm{O}$ pH encontrado variou entre 5,0 e 5,6. Nenhuma das temperaturas empregadas impactaram em alteração significativa no parâmetro de cor em relação às amostras liofilizadas. A capacidade de retenção de água do bagaço de malte seco foi maior que a de óleo. Os resultados indicam que sua secagem a $80^{\circ} \mathrm{C}$ é a melhor alternativa por manter características funcionais do resíduo e apresentar maior eficiência do processo em termos de tempo.

Palavras-Chaves: Bagaço de malte, Secagem, Valorização de resíduos, Sustentabilidade, Polifenóis.

\begin{abstract}
Brewery spent grain, considered the largest solid beer residue, is known to contain rich components that remain untapped during processing. In view of its high moisture content, this study evaluated the effects of drying on technological and nutritional characteristics important for the food industry. The influence of the drying process by natural convection, between 60 and $100{ }^{\circ} \mathrm{C}$, on the concentration of total polyphenols, flavonols, tartaric esters, compounds with antioxidant activity, $\mathrm{pH}$, color and water and oil retention capacity of malt bagasse was evaluated. from the lager production process. Freeze-dried samples were used for comparison. Dry samples at $60{ }^{\circ} \mathrm{C}$ and $80{ }^{\circ} \mathrm{C}$ showed a higher concentration of total polyphenols, flavonols, phenolic acids and compounds capable of eliminating free radicals. The $\mathrm{pH}$ found varied between 5.0 and 5.6. None of the temperatures employed impacted a significant change in the color parameter in relation to lyophilized samples. The water holding capacity of dry malt bagasse was greater than that of oil. The results indicate that drying at $80{ }^{\circ} \mathrm{C}$ is the best alternative for maintaining functional characteristics of the residue and presenting greater efficiency of the process in terms of time.

\footnotetext{
${ }^{1}$ Mestrado Profissional em Ambiente e Sustentabilidade, Universidade Estadual do Rio Grande do Sul, nutricao@educteutonia.com.br

${ }^{2}$ Bacharelado em Ciência e Tecnologia de Alimentos, Universidade Estadual do Rio Grande do Sul, gabidiersmann@hotmail.com

${ }^{3}$ Mestrado Profissional em Ambiente e Sustentabilidade, Universidade Estadual do Rio Grande do Sul, voltairesantanna@uergs.edu.br
} 
Keywords: Brewery spent grain, Drying, Waste recovery, Sustainability, Polyphenols.

\section{Introdução}

Responsável por gerar grande quantidade de resíduos, a atividade industrial de alimentos e bebidas é causa de preocupação, considerando que o destino incorreto destes subprodutos é um dos responsáveis pelas agressões ao ambiente. Para enfrentar este problema, diminuir sua geração seria o caminho mais racional, entretanto, o aumento da população global a uma velocidade exponencial e perdas que ocorrem nas diversas etapas da cadeia produtiva, demandam a implementação concomitante de práticas de redução e reutilização (MUSSATO, 2009; LYNCH et al., 2016).

Neste contexto, o bagaço de malte, considerado o maior resíduo sólido cervejeiro (MUSSATTO et al., 2006; XIROS; KRISTHAKOPOULOS, 2012) e formado no início do processo de produção de cerveja é conhecido por conter quantidades significativas de ricos componentes que permanecem inexplorados durante o processamento. Apesar de estar disponível em grandes volumes, seu destino usual tem sido a venda para elaboração de ração animal ou a própria doação para o mesmo fim. Consegue-se ter a dimensão do impacto ambiental que este setor pode provocar considerando que cada hectolitro (100 litros) de cerveja produzida gera entre 14 e $20 \mathrm{~kg}$ de bagaço, visto como sobras de processamento sem serventia ou valor econômico, e cujo montante pode chegar a $85 \%$ do total de resíduos gerados (MUSSATO et al., 2006; XIROS; KRISTHAKOPOULOS, 2012; LYNCH et al., 2016). Neste aspecto, a desidratação tem se apresentado como um caminho para melhorar a estabilidade microbiológica e bioquímica de resíduos da indústria alimentícia, permitindo estudos de viabilidade para ser utilizado como ingrediente nutricional para humanos.

O bagaço de cerveja tem se mostrado como importante fonte de polifenóis e fibras (MUSSATO et al., 2006; LYNCH et al., 2016), além de apresentar potencial de ser utilizado como ingrediente funcional na indústria de alimentos (SAURA-CALIXTO, 2011). Tendo em vista que o bagaço de malte proveniente da produção de cerveja apresenta alto teor de umidade levando o resíduo a um curto prazo de validade (MUSSATO et al., 2006; VALISH et al., 2011), este estudo tem o objetivo de avaliar os efeitos da secagem por convecção natural, na faixa de temperatura de 60 a $100^{\circ} \mathrm{C}$, sobre a cor, $\mathrm{pH}$, concentração de polifenóis totais, flavonóis, ésteres tartáricos, compostos com atividade antioxidante e capacidade de retenção de água e óleo, utilizando amostras liofilizadas como técnica de controle. Considerando que o malte tipo Pilsen é o mais utilizado, optou-se por estudar os efeitos da secagem exclusivamente sobre este que é o maior resíduo da indústria cervejeira. 


\section{Material e Métodos}

As matérias-primas utilizadas para a obtenção do bagaço de malte foram adquiridas em empresa local especializada em cervejas artesanais (Porto Alegre, RS, Brasil).

\section{Processo de produção do bagaço de malte}

Para o melhor controle da obtenção do bagaço de malte, os resíduos foram obtidos em laboratório, através do processo de brassagem de $2,3 \mathrm{Kg}$ de cevada malteada acrescidos de $7 \mathrm{~L}$ de água mineral. Tal mistura foi mantida a $42-45^{\circ} \mathrm{C}$ por $15 \mathrm{~min}, 52-55^{\circ} \mathrm{C}$ por $20 \mathrm{~min}$ e $62-65$ ${ }^{\circ} \mathrm{C}$ por mais $15 \mathrm{~min}$, sendo aquecida até atingir $80^{\circ} \mathrm{C}$ por $5 \mathrm{~min}$. Depois, procedeu-se a lavagem do produto com água corrente por $20 \mathrm{~min}$. Adicionou-se mais $8 \mathrm{~L}$ de água mineral à $80^{\circ} \mathrm{C}$ e repetiu-se a lavagem por $20 \mathrm{~min}$. $\mathrm{O}$ mosto foi filtrado. $\mathrm{O}$ resíduo inicial apresentou umidade inicial em torno de $72 \%( \pm 0,0063)$.

\section{Experimentos de secagem}

Imediatamente após sua obtenção, o bagaço de malte úmido foi submetido aos procedimentos de secagem, quando seu conteúdo foi dividido em duas amostras colocadas em estufa de ar estático (Solab SL100, São Paulo, Brasil). As amostras úmidas foram dispostas em camada única, em bandejas de metal de 12,5 cm de largura, 19,7 cm de comprimento e $1 \mathrm{~cm}$ de altura, em equipamento previamente aquecido. Cada amostra, separadamente, foi exposta às temperaturas de $60^{\circ} \mathrm{C}, 80^{\circ} \mathrm{C}$ e $100^{\circ} \mathrm{C}$. Mensurou-se a perda de massa a cada 30 min até atingir peso constante. Em seguida procedeu-se a moagem das amostras secas em processador industrial. O moedor foi higienizado com álcool $70 \%$ e deixado para secar naturalmente antes do preparo da amostra. Após este procedimento, as amostras foram peneiradas em malhas de 5 mm de abertura e transferidas para embalagens estéreis de polietileno escuro, dentro de uma capela laminar de fluxo. Amostras liofilizadas (Liotop L101, São Paulo, Brasil) foram utilizadas como controle. O teor de umidade das amostras, antes e após o experimento de secagem, foi determinado pelo método padrão AOAC ( 2001).

\section{Análise do pH}

Para determinar o $\mathrm{pH}$ das amostras, $1 \mathrm{~g}$ do resíduo seco foi diluído em 7,5 $\mathrm{mL}$ de água destilada fervida e resfriada por $1 \mathrm{~h}$, agitando suavemente a cada $15 \mathrm{~min}$. O sistema foi filtrado em papel Whatman $n^{\circ} 1$ e o extrato aquoso foi submetido à análise em pHmetro (Universal, modelo MT-610, São Paulo, Brasil), conforme AOAC (1990). 


\section{Análise de cor}

Os parâmetros CIELAB foram determinados usando iluminação difusa D-65 de um colorímetro Minolta Chroma CR-400 (Konica Minolta). O instrumento foi calibrado com uma placa branca padrão. Os parâmetros medidos foram $L^{*}$ (luminosidade), $a^{*}$ (vermelhidão) e $b^{*}$ (amarelo). São coordenadas cromáticas demonstradas por um diagrama de cores, cujos parâmetros foram definidos pela Comissão Internacional de Iluminação (CIE) e chamado de espaço CIELab.

\section{Análise de polifenóis}

Os extratos foram preparados misturando-se $1 \mathrm{~g}$ de matéria seca em pó do bagaço de malte em $50 \mathrm{~mL}$ de solução de etanol $50 \%$ (v:v), mantendo o sistema a $60{ }^{\circ} \mathrm{C}$ por 30 min para posterior análise (SANT’ANNA, 2013). Em seguida, os extratos foram filtrados em papel filtro Whatman $\mathrm{n}^{\circ} 1$. As extrações foram realizadas em duplicata.

A concentração de polifenóis totais foi determinada pelo método de Folin-Ciocalteau descrito por Singleton e Rossi (1965), onde alíquotas de $40 \mu \mathrm{L}$ dos extratos foram misturados com 3,2 mL de água destilada e $200 \mu \mathrm{L}$ de solução de carbonato de sódio $20 \%$ (m/v). Após 15 min no escuro, foram adicionados $600 \mathrm{~mL}$ de solução do reagente de Folin-Ciocalteau e o sistema mantido à temperatura ambiente no escuro por $90 \mathrm{~min}$. A absorbância foi medida a 765 nm (SINGLETON; ROSSI, 1965) pelo espectrofotômetro UV-1600 (Pró-Análise, Brasil). Para quantificação foi empregada uma curva padrão com solução de ácido gálico nas seguintes concentrações: 200, 300, 500, 600, 700, 800, 900, 1000 mg/L. Foi calculado um coeficiente de correlação $\left(\mathrm{R}^{2}\right)$, resultando $\mathrm{R}^{2}=0,9902$. O teor de polifenóis totais foi expresso em $\mathrm{mg}$ equivalente de Ácido Gálico (EAG)/100g de bagaço seco. O reagente Folin-Ciocalteau e o ácido gálico foram obtidos na Vetec Química Fina (Duque de Caxias, Brasil). Os produtos químicos restantes foram comprados da Neon (Suzano, Brasil).

A concentração de flavonóis totais foi determinada usando o método descrito por Mazza e colaboradores (1999). Amostras de $250 \mu \mathrm{L}$ de extrato foram misturados com $250 \mu \mathrm{L}$ de $\mathrm{HCl}$ $0,1 \%$ em etanol $95 \%$ e $4,5 \mathrm{~mL}$ de $\mathrm{HCl}$ a $2 \%$. A mistura foi submetida a um agitador mecânico e colocada em repouso no escuro por $15 \mathrm{~min}$. Posteriormente foi realizada a leitura no espectro e comparada a uma amostra de água destilada. A absorbância a $360 \mathrm{~nm}$ foi então mensurada. Para quantificação foi empregada uma curva padrão com solução de epicatequina nas concentrações entre 10 e $200 \mathrm{mg} / \mathrm{L}$. Foi calculado um coeficiente de correlação $\left(\mathrm{R}^{2}\right)$, resultando 
$\mathrm{R}^{2}=0,9976$. O teor de flavonóis foi expresso em mg equivalente de epicatequina (EE)/100g de bagaço seco.

A concentração de ácidos fenólicos foi determinada usando o método descrito por Mazza e colaboradores (1999). Amostras de $250 \mu \mathrm{L}$ de extrato foram misturados com $250 \mu \mathrm{L}$ de $\mathrm{HCl}$ 0,1\% em etanol 95\%. Depois, $4,5 \mathrm{~mL}$ de $\mathrm{HCl}$ a $2 \%$. A mistura foi submetida a um agitador mecânico e colocada em repouso no escuro por 15 min. Posteriormente realizou-se a leitura no espectro e comparou-se a uma amostra de água destilada. A absorbância a $320 \mathrm{~nm}$ foi então mensurada. Para quantificação foi empregada uma curva padrão com solução de ácido caféico nas concentrações entre 10 e 200 mg/L. Foi calculado um coeficiente de correlação $\left(\mathrm{R}^{2}\right)$, resultando $\mathrm{R}^{2}=0,9936$. O teor de ácidos fenólicos foi expresso em mg equivalente de ácido caféico (ACE)/100g de bagaço seco.

\section{Atividade antioxidante}

A atividade antioxidante do bagaço seco foi avaliada pelos métodos de sequestro do radical DPPH (1,1-difenil-2-picrilhidrazil) e eliminação do radical ABTS (ácido 3etilbenzotiazolina-6-sulfônico) gerado durante o ensaio. A capacidade de sequestrar o radical DPPH, segundo método descrito por Brand-Williams et al. (1995), se dá pela mistura de alíquotas do extrato das amostras solução de DPPH (Sigma Aldrich, EUA) em metanol. Após agitação, os tubos foram deixados em repouso ao abrigo da luz por aproximadamente 15 a 20 min e a absorbância foi medida a $515 \mathrm{~nm}$. A determinação da atividade de eliminação do radical ABTS, conforme Re et al. (1999), envolve a geração do cromóforo do radical ABTS por oxidação do ABTS com persulfato de potássio (Neon (Suzano, Brasil)). O radical catiônico $\operatorname{ABTS}^{\bullet+}$ (Sigma Aldrich, EUA) foi produzido por reação da solução de $7 \mathrm{mmol} / \mathrm{L}$ ABTS com $140 \mathrm{mmol} / \mathrm{L}$ de persulfato de potássio, a qual permaneceu no escuro por 16h, à temperatura ambiente antes do uso. Para o ensaio, a solução foi diluída com etanol até uma absorbância de 0,7 a $734 \mathrm{~nm}$. Uma alíquota de $30 \mu \mathrm{L}$ de extrato foi misturada com $1 \mathrm{~mL}$ da solução e, uma leitura de absorbância $(734 \mathrm{~nm})$ foi realizada após 6 minutos. Água destilada, em vez de amostra, foi usada para comparação. A capacidade de sequestrar o radical foi expressa em percentual, calculada em relação ao controle (sem antioxidante). Os resultados foram expressos como: atividade de eliminação $(\%)=\left[1-\left(\mathrm{A} / \mathrm{A}_{0}\right)\right] \times 100$, onde $\mathrm{A}$ é a absorbância do teste, e $\mathrm{A}_{0}$ é a absorbância do controle. 


\section{Capacidade de retenção de água e de óleo}

A capacidade de retenção de água das amostras de bagaço de malte seco foi analisada pela manutenção de $0,5 \mathrm{~g}$ da mistura em $10 \mathrm{~mL}$ de água destilada por 16 horas de acordo com Rosell et al. (2009), com modificações. O sistema foi então centrifugado à temperatura ambiente e rotação de $140 \mathrm{~g}$ e a parte líquida pesada. A capacidade de retenção de água foi calculada pela a diferença de líquido adicionado e não absorvido, descontando-se a umidade inicial do material.

A capacidade de absorção de óleo foi analisada pela manutenção da mesma massa de amostra mantida em contato com $50 \mathrm{~mL}$ de azeite de oliva extra virgem por 24 horas. Os sistemas foram centrifugados a $140 \mathrm{~g}$ e diferença e a fração não absorvida foram pesadas em balança analítica, conforme Rosell et al. (2009).

\section{Análise estatística}

As experiências foram conduzidas em triplicata e as médias de dois experimentos independentes foram calculadas. Os valores obtidos foram comparados por Análise de Variância (ANOVA), pelo software Excel 2013 e tratadas pelo teste de Tukey, pelo Statistica 10.0. As diferenças foram consideradas estatisticamente significativas quando $p<0,05$.

\section{Resultados e Discussão}

\section{Polifenóis totais, flavonóis, ésteres tartáricos e atividade antioxidante}

Extratos de malte e cevada caracterizam-se pelo alto teor de flavonóis e ácidos fenólicos, os quais lhe conferem potente atividade antioxidante (BARBOSA-PEREIRA et al., 2014).

$\mathrm{O}$ efeito das diferentes temperaturas de secagem sobre a concentração de compostos bioativos e a atividade antioxidante do bagaço de malte seco encontrados neste estudo estão demonstrados na Tabela 1.

Entre as amostras secas em diferentes temperaturas, àquelas submetidas à $60{ }^{\circ} \mathrm{C}$ apresentaram maior concentração de polifenóis totais, flavonóis, ácidos fenólicos e compostos com capacidade de eliminar radicais ABTS(ácido 3-etilbenzotiazolina-6-sulfônico) ao final do processo. Em comparação com as amostras liofilizadas, àquelas expostas a procedimentos de secagem a 60 e $80{ }^{\circ} \mathrm{C}$ apresentaram quantidades semelhantes $(p>0,05)$ de polifenóis totais e flavonóis após o processo, resultado semelhante encontrado por Hii e colaboradores (2009), em amostras secas de cacau. Contudo, a $100{ }^{\circ} \mathrm{C}$, o bagaço seco apresentou quantidades significativamente $(p<0,05)$ menores destas substâncias. Altas temperaturas podem impactar a 
degradação de vários compostos, incluindo polifenóis (LARRAURI et al.,1997; WANG; XU, 2007).

Tabela 1. Influência de diferentes temperaturas de secagem sobre a concentração de polifenóis totais, flavonóis, ésteres tartáricos e compostos com atividade antioxidante no bagaço de Malte

\begin{tabular}{lcccc}
\hline & $100^{\circ} \mathrm{C}$ & $80^{\circ} \mathrm{C}$ & $60^{\circ} \mathrm{C}$ & Liofilizado \\
\hline $\begin{array}{l}\text { Polifenóis totais } \\
(\mathrm{mg} \text { GAE/100g) }\end{array}$ & $7601,402 \pm 759,75^{\mathrm{b}}$ & $11944,962 \pm 834,67^{\mathrm{a}}$ & $12210,499 \pm 1500,18^{\mathrm{a}}$ & $9515,595 \pm 1528,01^{\mathrm{a}}$ \\
$\begin{array}{l}\text { Flavonóis } \\
(\mathrm{mg} \mathrm{EE} / 100 \mathrm{~g})\end{array}$ & $2620,742 \pm 260,30^{\mathrm{b}}$ & $3395,449 \pm 241,19^{\mathrm{a}}$ & $3604,209 \pm 327,89^{\mathrm{a}}$ & $3618,775 \pm 397,71^{\mathrm{a}}$ \\
$\begin{array}{l}\text { Ésteres tartáricos } \\
(\mathrm{mg} \text { CA/100g) }\end{array}$ & $1440,029 \pm 226,55^{\mathrm{b}}$ & $1423,463 \pm 234,33^{\mathrm{b}}$ & $1538,401 \pm 139,80^{\mathrm{b}}$ & $3601,620 \pm 157,90^{\mathrm{a}}$ \\
DPPH (\%) & $6,036 \pm 0,019^{\mathrm{c}}$ & $10,905 \pm 1,549^{\mathrm{b}}$ & $10,603 \pm 0,931^{\mathrm{b}}$ & $16,183 \pm 1,853^{\mathrm{a}}$ \\
ABTS (\%) & $48,075 \pm 0,53^{\mathrm{b}}$ & $50,625 \pm 0,39^{\mathrm{b}}$ & $60,225 \pm 3,85^{\mathrm{a}}$ & $68,875 \pm 0,39^{\mathrm{a}}$ \\
\hline
\end{tabular}

$\overline{\mathrm{a}, \mathrm{b}, \mathrm{c}}$ letras diferentes na mesma linha indicam diferença significativa $(p<0,05)$.

Fonte: Autora (2019).

Barbosa-Pereira e colaboradores (2014), comprovaram em sua pesquisa que extratos brutos de compostos fenólicos com alta atividade antioxidante pode ser obtida a partir dos resíduos de cervejarias, e que podem ser usados como fonte barata de compostos. Moreira e colaboradores (2013), observaram que os bagaços de maltes pilsen, melano, melano 80 e cararé, apresentaram maiores quantidades de compostos fenólicos quando comparados ao chocolate e ao bagaço preto $(19,5 \pm 0,6$ e 16,2 \pm 0,6 $\mathrm{mg}$ GAE /g db para os tipos claro e escuro, respectivamente). Samaras e colaboradores (2005), descobriram que a catequina e o ácido ferúlico eram os compostos fenólicos mais abundantes nos maltes mortos e torrados, e que os ácidos ferúlicos eram altamente sensíveis à degradação durante o tratamento de assados.

Os resultados referentes a quantidade de ácidos fenólicos encontrados nas amostras de BM secas a 60,80 e $100{ }^{\circ} \mathrm{C}$ apresentaram comportamento semelhante entre si $(p>0,05)$, porém antagônico às amostras liofilizadas, ou seja, em qualquer uma das três temperaturas de secagem houve perda maior de ésteres tartáricos em comparação às amostras que passaram por liofilização. Hii e colaboradores (2009), da mesma forma, demonstraram em seu estudo, não haver diferença significativa no conteúdo fenólico para amostras secas a 60 e $80^{\circ} \mathrm{C}$.

A capacidade de eliminação do radical ABTS (ácido 3-etilbenzotiazolina-6-sulfônico) analisada no bagaço de malte seco a $60{ }^{\circ} \mathrm{C}$ demonstrou semelhança $(p>0,05)$ ao resultado encontrado na amostra liofilizada. Contudo, resultados diferentes foram constatados ao desidratar as amostras à 80 e $100{ }^{\circ} \mathrm{C}$. Após serem submetidas a estas temperaturas, verificouse perda significativa $(p<0,05)$ da capacidade das amostras de bagaço de malte de eliminar o radical ABTS (ácido 3-etilbenzotiazolina-6-sulfônico). 
A capacidade de sequestrar o radical DPPH (1,1-difenil-2-picrilhidrazil), analisada no bagaço de malte seco a $60{ }^{\circ} \mathrm{C}$ demonstrou semelhança $(p>0,05)$ ao resultado encontrado na amostra seca a $80{ }^{\circ} \mathrm{C}$. Contudo, resultados diferentes foram constatados ao desidratar as amostras à $100{ }^{\circ} \mathrm{C}$, cujos resultados após o procedimento, demonstraram perda significativa $(p<0,05)$ de tal capacidade. Sant'Anna (2013) ao analisarem efeitos da secagem sobre o bagaço de uva Vitis labrusca $c v$. "Isabel" demonstraram que compostos fenólicos totais e compostos com capacidade de eliminação de radicais DPPH permaneceram estáveis, embora compostos com capacidade de eliminação de radicais ABTS mostraram-se suscetíveis a degradação.

pH

$\mathrm{O}$ efeito da secagem sobre o $\mathrm{pH}$ do resíduo seco está representado na Figura 1. Os resultados mostram que o bagaço de cerveja seco apresenta $\mathrm{pH}$ ácido entre 5,0 e 5,6, o que pode ser importante para sua estabilidade microbiológica durante o período de armazenamento. Larsson e Sanderberg (1995) observaram valores semelhantes de $\mathrm{pH}$ para aveias malteadas, indicando maior estabilidade microbiana devido ao $\mathrm{pH}$ ácido das amostras. A secagem do resíduo à 60 e $80^{\circ} \mathrm{C}$ não impactou em alteração significativa $(p>0,05)$ do $\mathrm{pH}$ das amostras em comparação àquelas liofilizadas.

Figura 1. Efeito dos tratamento de secagem sobre o $\mathrm{pH}$ de bagaço de cerveja

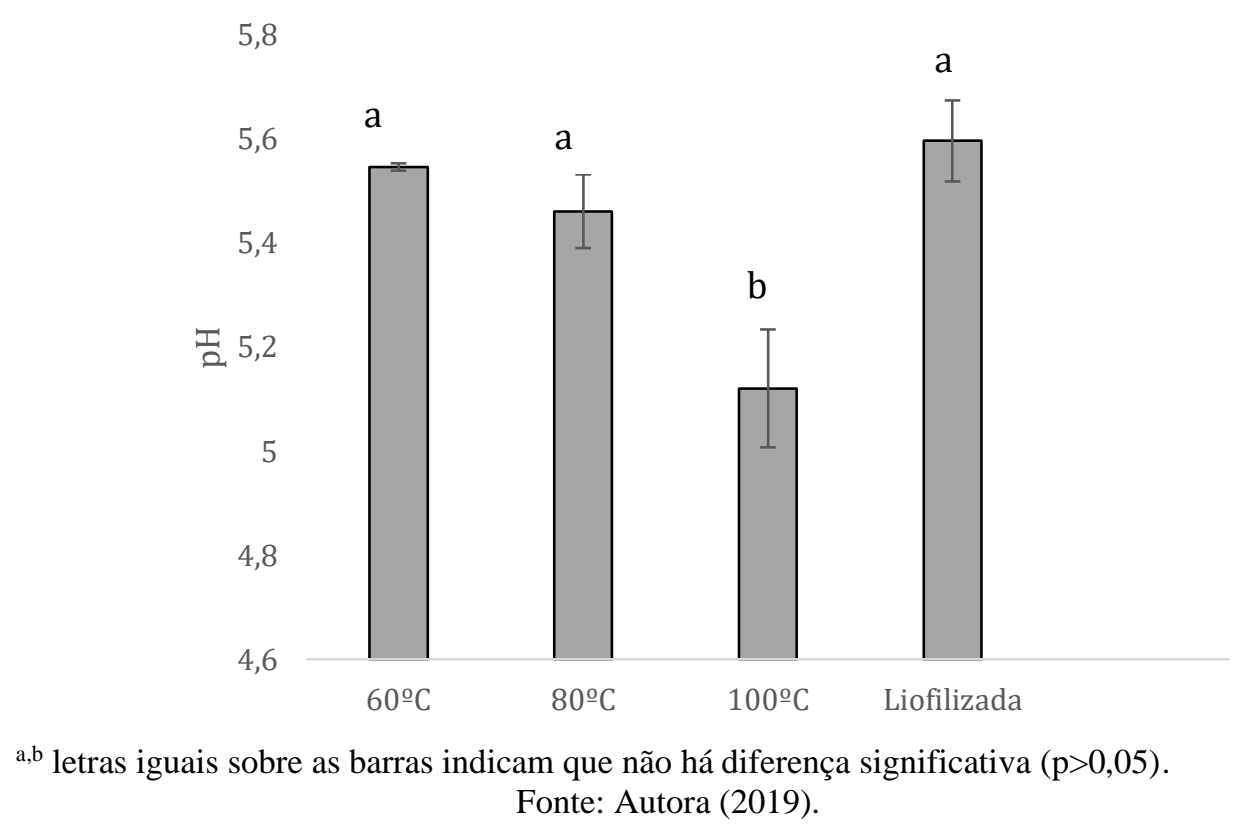

$\mathrm{Na}$ utilização de temperatura de $100{ }^{\circ} \mathrm{C}$ para a secagem, obervou-se redução significativa $(p<0,05)$ nesta propriedade. A redução do $\mathrm{pH}$ pode estar relacionada à hidrólise de 
lipídeos, produzindo ácidos graxos ou hidrólise protéica, produzindo aminoácidos ou produtos intermediários com maior acidez (MIRANDA; EL-DASH, 2002).

\section{Análise de cor}

Os resultados da análise de cores, de acordo com a metodologia CIELAB, estão demonstrados na Tabela 2.

Tabela 2. Análise de cor do bagaço de cerveja seco em diversas temperaturas e liofilizado

\begin{tabular}{|c|c|c|c|}
\hline & $L^{*}$ & $a^{*}$ & $b^{*}$ \\
\hline Liofilizado & $40,18 \pm 0,98^{\mathrm{a}}$ & $5,63 \pm 0,02^{\mathrm{a}}$ & $18,46 \pm 0,38^{\mathrm{a}}$ \\
\hline $100^{\circ} \mathrm{C}$ & $42,02 \pm 0,94^{\mathrm{a}}$ & $4,86 \pm 0,10^{\mathrm{a}}$ & $19,52 \pm 0,28^{\mathrm{a}}$ \\
\hline $80^{\circ} \mathrm{C}$ & $42,72 \pm 0,05^{\mathrm{a}}$ & $5,05 \pm 0,24^{\mathrm{a}}$ & $20,04 \pm 0,05^{\mathrm{a}}$ \\
\hline $60^{\circ} \mathrm{C}$ & $40,22 \pm 1,02^{\mathrm{a}}$ & $4,92 \pm 0,11^{\mathrm{a}}$ & $18,67 \pm 0,47^{\mathrm{a}}$ \\
\hline
\end{tabular}

Os resultados mostram que não houve diferença significativa $(p>0,05)$ para o parâmetro $L^{*}$, indicando que as amostras apresentam grau de coloração escura semelhante. Resultados semelhantes foram encontrados sobre o impacto da secagem em forno de ar estático para os parâmetros $a^{*}$ e $b^{*}$, em que as temperaturas empregadas não impactaram em alteração significativa $(p>0,05)$ no parâmetro de cor em relação às amostras liofilizadas, demonstrando ser uma forma mais econômica de desidratar o bagaço de malte sem alterar sua cor característica.

O fato do grau de coloração das amostras não apresentar diferença significativa em nenhum dos parâmetros avaliados pode estar ligado ao efeito que o tratamento térmico tem durante a formação das cores dos maltes, sendo os escuros obtidos pela utilização de tostadores a temperaturas acima de $200{ }^{\circ} \mathrm{C}$. Para obtenção de maltes claros, utilizam-se temperaturas amenas durante o processo de secagem dos grãos germinados, como as utilizadas neste experimento, com o objetivo de remover umidade e conservar enzimas que atuam de forma imprescindível na conversão do amido em açúcares solúveis responsáveis, entre outras características, pela cor (LI et al., 2014). Larrauri e colaboradores (1998), avaliaram a influência de temperaturas entre 60 e $140^{\circ} \mathrm{C}$ na degradação da cor após secagem de bagaço de uva e concluíram que a intensidade da cor vermelha não diminuiu com o tratamento entre 60 e $100{ }^{\circ} \mathrm{C}$, porém o mesmo não ocorreu com a luminosidade em relação à amostra liofilizada. 


\section{Capacidade retenção de água e óleo}

Os resultados para a capacidade de absorção de água e óleo estão representados na Figura 2. As amostras de bagaço de malte liofilizadas apresentaram capacidade significativamente superior $(p<0,05)$ de retenção de água quando comparadas à secagem com equipamentos convencionais. Santana e Oliveira (2005) observaram resultados semelhantes em sua pesquisa com subproduto do maracujá em pó. Ainda em relação a análise da capacidade absorção de água, Collar e colaboradores (2007) e Ferreira e colaboradores (2013), também observaram que fibra comercial de maçã, bambu, aveia e farinhas de vegetais apresentaram capacidade de retenção de água com valores semelhantes aos encontrados para o resíduo de cerveja no presente trabalho. A Figura 2 mostra ainda que a capacidade de absorção de água pelo bagaço de malte seco em estufa de ar estático, também foi maior que a de óleo. Este mesmo resultado foi constatado por Huige (1994) em sua pesquisa com farinha de bagaço de malte. Além de apresentar alto teor de fibras alimentares, o estudo demonstrou que o subproduto tem grande capacidade de absorção de água e reduzida habilidade de absorção de gordura. Isso possivelmente se deve ao fato do resíduo ser rico em monossacarídeos e polissacarídeos e seus derivados.

Figura 2. Efeito da secagem sobre a capacidade de absorção de água (barras pretas) e óleo (barras cinzas) de bagaço de malte

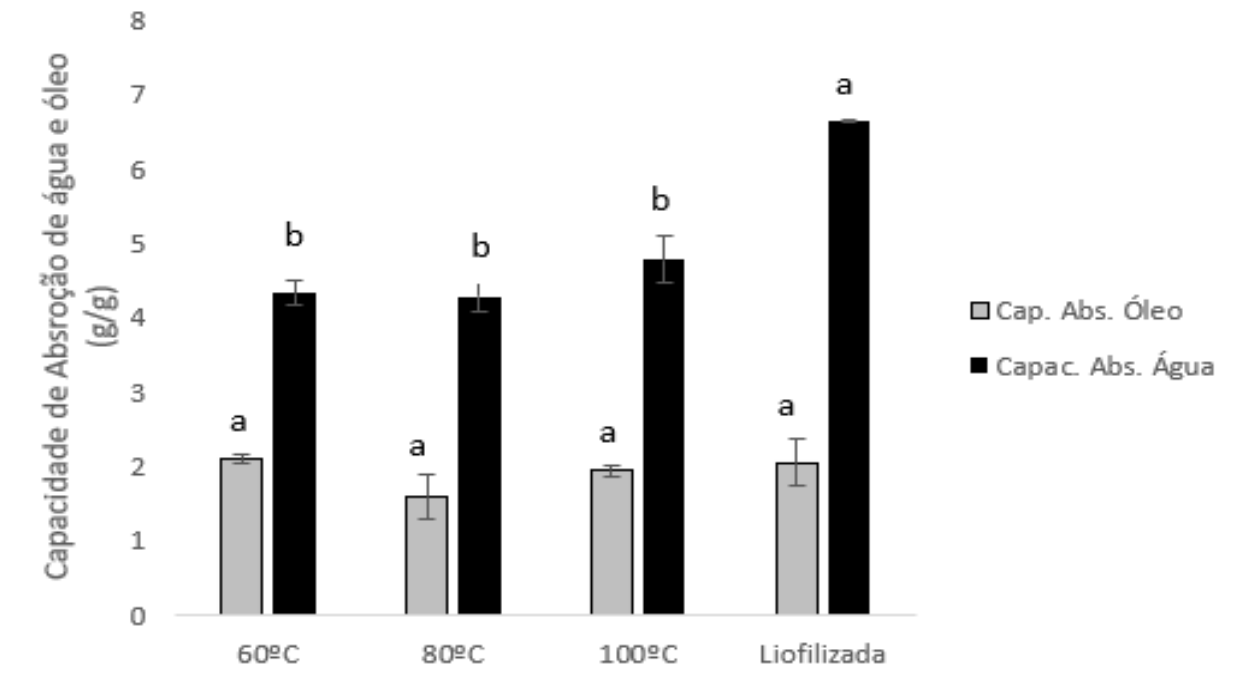

${ }^{\mathrm{a}, \mathrm{b}}$ letras diferentes sobre barras da mesma cor indicam diferença significativa $(\mathrm{p}<0,05)$ Fonte: Autora (2019).

Sabe-se que o grão de cevada tem um nível de beta-glucanos que é muito maior do que o encontrado em outros grãos (NEWMAN et al., 1989; MCINTOSH et al., 1991). Os betaglucanos, segundo Cavallero et al. (2002), são uma espécie de fibra alimentar solúvel que, uma vez na presença de água, avoluma-se para formar soluções viscosas. 
Em relação ao efeito dos diferentes tratamentos de secagem sobre a propriedade de absorção de água do bagaço de malte, observou-se diferença significativa $(p<0,05)$ entre todos eles e a técnica de liofilização, a qual demonstrou maior parâmetro. Santana e Oliveira (2005), da mesma forma, observaram que o subproduto liofilizado do maracujá em pó apresentou maior índice de absorção de água nas amostras liofilizadas do que as submetidas à secagem por ar quente.

Em relação à capacidade de absorção de óleo, observa-se que não houve diferença significativa $(p>0,05)$ para os diferentes tratamentos testados. Ferreira e colaboradores (2013), observaram valores semelhantes de capacidade de absorção de óleo para farinhas de frutas e vegetais em seu estudo.

\section{Conclusões}

O maior resíduo sólido cervejeiro demonstra, através deste estudo, ter qualificação para ser uma fonte promissora de antioxidantes naturais, podendo ser uma alternativa para substituir os antioxidantes sintéticos atualmente usados na indústria de alimentos. Além disso, demonstra capacidade de manter outras qualidades físico-químicas que lhe habilitam como opção econômica, por ser facilmente obtido através de procedimentos comuns de secagem.

Os resultados do presente estudo indicam que a utilização de temperaturas de secagem na faixa de $60^{\circ} \mathrm{C}-80^{\circ} \mathrm{C}$ não impactam na concentração de diferentes classes de polifenóis e compostos com diferentes atividades antioxidantes extraíveis da matriz alimentar. Nessa faixa de temperatura, os resultados mostram que a técnica de liofilização não implica em melhor eficiência. Mesmo comportamento é observado em relação à cor e pH e capacidade de absorção de óleo. A utilização de temperaturas de $100{ }^{\circ} \mathrm{C}$, contudo, implica em redução de compostos bioativos. Devido ao menor tempo de processamento, os resultados do presente trabalho indicam a utilização de temperaturas de secagem de até $80^{\circ} \mathrm{C}$, apesar de mais estudos de eficiência de processo serem necessários.

\section{Referências}

ASSOCIATION OF OFFICIAL ANALYTICAL CHEMISTS. Official methods of analysis of the Association of the Analytical Chemistry. 15. ed. Arlington, 1990. ASSOCIATION OF OFFICIAL ANALYTICAL CHEMISTS. Official methods of analysis of the Association of the Analytical Chemistry. 17. ed. Arlington, 2001. 
BARBOSA-PEREIRA, L.; BILBAO, A.; VILCHES, P.; ANGULO, I.; LLUIS, J.; FITÉ, B.; PASEIRO-LOSADA, P.; CRUZ, J.M. Phenolic profile and antioxidant properties of a crude extract obtained from a brewery waste stream. Food Research International, v. 51, n. 2, p. 663-669, 2014.

BRAND-WILLIAMS, W.; CUVELIER, M.; BERSET, C. Use of a free radical method to evaluate antioxidant activity. LWT-Food Science and Technology, v. 28, p. 25-30, 1995.

CAVAllERO, A.; EMPILlI, S.; BRIGHENTI, F.; STANCA, A. M. High (1-3,1-4)-b-glucan barley fractions in bread making and their effects on human glycemic response. Journal of Cereal Science, v.36, p.59-66, 2002.

COLLAR, C.; SANTOS, E.; ROSELL, C.M. Assessment of the rheological profile of fibreenriched bread doughs by response surface methodology. Journal of Food Engineering, v.78, n.3, p. 820-826, 2007.

DOBRZANSKI, J; DIAS, L.F; AYALA, L.A.C. Característica e utilização do bagaço de cerveja em panificação. Anais da IV Semana de Tecnologia em Alimentos, 4, 2008, Ponta Grossa. Paraná: UTFPR. v.2, n.7, 2008.

FERREIRA, M. S. L.; SANTOS, M. C. P.; MORO, T. M. A.; BASTO, G. J.; ANDRADE, R. M. S.; GONÇALVES, É. C. B. A. Formulation and characterization of functional foods based on fruit and vegetable residue flour. Journal of Food Science and Technology, v.52, n.2, p.822-830, 2013.

HII, C. L.; LAW, C. L.; CLOKE, M.; SUZANNAH, S. Thin layer drying kinetics of cocoa and dried product quality. Biosystem Engineering, v.102, p.153-161, 2009.

HUIGE, N.J. Brewery by-products and effluents. In: Hardwick, W.A. (Ed.). Handbook of Brewing. New York: Marcel Dekker, 1994, p. 501-550.

LARRAURI, J. A.; RUPÉREZ, P.; BORROTO, B.; SAURA-CALIXTO, F. Effect of drying temperature on the stability of polyphenols and antioxidant activity of red grape pomace peels. Journal of Agricultural and Food Chemistry, v.45, p.1390-1393, 1997. 
LARSSON, M.; SANDERBERG, A.S. Malting of oats in a pilot-process. Effects of heat treatment storage and soaking conditions on phytate reduction. Journal of Cereal Science, v.21, p.87-95, 1995.

LI, X.; JIN, Z.; GAO, F.; LU, J.; CAI, G.; DONG, J.; YU, J.; YANG, M. Comparative Proteomic Analysis of Dan'er Malts Produced from Distinct Malting Processes by TwoDimensional Fluorescence Difference in Gel Electrophoresis (2D-DIGE). Journal of Agricultural and Food Chemistry, v.62, n.38, p.9310-9316, 2014.

LYNCH, K. M.; STEFFEN, E.J.; ARENDT, E. K. Brewers' spent grain: a review with an emphasis on food and health Inst. Brew. Journal of the Institute of Brewing, v.122, p 553$568,2016$.

MAZZA, G.; FUKUMOTO, L.; DELAQUIS, P.; GIRARD, B.; EWERT, B. Anthocyanins, phenolics, and color of Cabernet Franc, Merlot, and Pinot Noir wines from British Columbia. Journal of Agricultural and Food Chemistry, v.47, n.10, p.4009-4017, 1999.

MCINTOSH, G. H.; WHYTE, J.; MCARTHUR, R.; NESTEL, P. J. Barley and wheat foods: influence on plasma cholesterol concentrations in hypercholesterolemic men. American Journal of Clinical Nutrition, v.53, n.5, p.1205-1209, 1991.

MIRANDA, M. Z. de; EL-DASH, A. Farinha integral de trigo germinado: Características nutricionais e estabilidade ao armazenamento. Ciência e Tecnologia de Alimentos, v.22, n.3, p.216-223, 2002.

MOREIRA, M. M., MORAIS, S., CARVALHO, D. O., BARROS, A. A., DELERUE-MATOS, C., GUIDO, L. F. Brewer's spent grain from different types of malt: Evaluation of the antioxidant activity and identification of the major phenolic compounds. Food Research International, v.54, p.382-388, 2013.

MUSSATTO, S. I., DRAGONE, G., ROBERTO, I. C. Brewer's spent grain: generation, characteristics and potential applications. Journal of Cereal Science, v.43, p.1-14, 2006. 
MUSSATTO, S. I. Biotechnological potential of brewing industry by-products. In: Singh nee' Nigam, P., Pandey, A. (eds.) Biotechnology for Agro-Industrial Residues Utilisation, p. 314-326. Netherlands: Springer Science+Business Media BV, 2009.

NEWMAN, R. K.; LEWIS, S. E.; NEWMAN, C. W.; BOIK, R. J.; RAMAGE, R. T. Hypochoesterolemic effect of barley foods on healthy men. Nutritional Reports International, v.39, p.749-760, 1989.

OliveirA FILHO, J. F. Extração de Corante Natural a Partir do Resíduo da Uva. Orientadora: Sousa, Magna Angélica dos Santos Bezerra. 2017. 45f. Trabalho de Conclusão de Curso (Graduação em Engenharia Química) - Departamento de Engenharia Química, Universidade Federal do Rio Grande do Norte, Natal, 2017.

RE, R.; PELLEGRINI, N.; PROTEGGENTE, A.; PANALA, A.; YANG, M.; RICE-EVANS, C. Antioxidant activity applying an improved ABTS radical cation decolorization assay. Free Radical Biology and Medicine, v.26, p.1231-1237, 1999.

ROSELL, C. M.; SANTOS, E.; COLLAR, C. Physico-chemical properties of commercial fibres from different sources: A comparative approach. Food Research International, v.42, p.176-184, 2009.

SAMARAS, T. S.; CAMBURN, P. A.; CHANDRA, S.; GORDON, M. H.; AMES, J. M. Antioxidant Properties of Kilned and Roasted Malts. Journal of Agricultural and Food Chemistry, v.53, p.8068-8074, 2005.

SANT'ANNA, V. Concentração de suco de uva por osmose direta e estudo tecnológico para o aproveitamento do bagaço. Orientadora: Tessaro, Isabel Cristina. 2013. 177f. Tese de Doutorado (Programa de Pós-Graduação em Engenharia Química) - Departamento de Engenharia Química, Universidade Federal do Rio Grande do Sul, Porto Alegre, 2013.

SANTANA, A. F.; OLIVEIRA, L. F. Aproveitamento da casca de melancia (Curcubita citrullus, shrad) na produção artesanal de doces alternativos. Alimentos e Nutrição Araraquara, v.6, n.4, p.363-368, 2005. 
BAGATINI, L.; AZEVEDO, G. D.; SANT’ANNA, V.

SAURA-CALIXTO, F. Dietary Fiber as a Carrier of Dietary Antioxidants: An Essential Physiological Function. Journal of Agricultural and Food Chemistry, v.59, p 43-49, 2011.

SINGLETON, V. L; ROSSI, J. A. Colorimetry of total phenolics with phosphomolybdicphosphotungstic acid reagents. American Journal of Enology and Viticulture, v.20, p.144$158,1965$.

VASHISHT, T.; SINGH, R. K.; PEGG, R. B. Effects of drying on the phenolics content and antioxidant activity of muscadine pomace. LWT - Food Science and Technology, v.44, p.1649-1657, 2011.

XIROS, C.; CHRISTAKOPOULOS, P. Biotechnological Potential of Brewers Spent Grain and its Recent Applications. Waste and Biomass Valorization, v.3, n.2, p.213-232, 2012.

WANG, W.-D.; XU, S.-Y. Degradation kinetics of anthocyanins in blackberry juice and concentrate. Journal of Food Engineering, v.82, n.3, p.271-275, 2007. 